\title{
G76.188+0.098: a newly born massive binary star ${ }^{\star}$
}

\author{
F. Comerón ${ }^{1}$, A. Pasquali ${ }^{2}$, and J. Torra ${ }^{3}$ \\ 1 European Southern Observatory, Karl-Schwarzschild-Strasse 2, 85748 Garching, Germany \\ e-mail: fcomeron@eso.org \\ 2 Max-Planck-Institut für Astronomie, Königstuhl 17, 69117 Heidelberg, Germany \\ e-mail: pasquali@mpia-hd.mpg.de \\ 3 Departament d'Astronomia i Meteorologia, Universitat de Barcelona, 08028 Barcelona, Spain \\ e-mail: jordi@am.ub.es
}

Received 12 February 2006 / Accepted 4 July 2006

\begin{abstract}
Context. We present observations of G76.188+0.098, an object classified in the literature as an ultracompact HII region (UCHIIR) based on its far-infrared and radio continuum characteristics.

Aims. To investigate the nature of this object on the basis of the combination of radio observations existing in the literature and the new observations in the near-infrared presented here, with particular attention to the direct detection of the central ionizing source. Methods. Near-infrared narrow-band imaging and low resolution spectroscopy in the $2 \mu \mathrm{m}$ region.

Results. The new observations clearly reveal the central source, which turns out to be a resolved binary system obscured by $A_{K} \simeq 4.4 \mathrm{mag}$. A general agreement is found between the morphology of the region as outlined by narrow-band, near-infrared imaging, and existing VLA radio continuum maps. Indirect evidence based on the low HeI $(2.058 \mu \mathrm{m}) / \mathrm{Br} \gamma$ intensity ratio of the compact nebula suggests that the binary system is composed of two stars with spectral types O9 and B0 at a distance of $\sim 2.4 \mathrm{kpc}$, somewhat closer to the Sun than commonly assumed in the literature.

Conclusions. The size of the central cavity of the compact nebula, the low electron densities derived from radio continuum measurements, and the moderate extinction towards the central source by UCHIIR standards suggest that G76.188+0.098 is a very young massive binary observed near the end of the embedded, ultracompact phase, adding one example to the rare cases in which the central source of an ultracompact HII region is detected.
\end{abstract}

Key words. ISM: individual objects: G76.188+0.098 - stars: early-type - binaries: general - HII region

\section{Introduction}

Observations of the earliest stages of massive stellar evolution are challenging. The first manifestation of the existence of a newly formed star as massive as several tens of a solar mass is usually found in the far-infrared and radio domains, when the star begins ionizing the dense remnants of its prestellar core in which it is still deeply embedded. The resulting object, an UltraCompact HII Region (UCHIIR; see Churchwell 2002 for a recent review), often has a radio spectrum characterized by a high turnover frequency (typically around $\sim 5 \mathrm{GHz}$ ) due to the large emission measure $\left(E M>10^{7} \mathrm{~cm}^{-6} \mathrm{pc}\right)$, which is in turn due to the very high volume density of ionized gas $\left(n_{\mathrm{H}}>10^{4} \mathrm{~cm}^{-3}\right)$. The frequent detection of $\mathrm{CS}$ and $\mathrm{NH}_{3}$ cores in their direction indicates that UCHIIRs are associated with dense molecular gas. Their physical sizes are small, typically $<0.1 \mathrm{pc}$, implying that UCHIIRs are dynamically unevolved objects. Models can reproduce the spectral energy distribution of UCHIIRs by means of an optically and geometrically thick dust cocoon with a small central cavity (Faison et al. 1998, and references therein), rendering the central star virtually invisible even in the near-infrared. However, observations have thus far revealed a handful of cases in which the photosphere of the central star can be directly

* Based on observations collected at the Centro Astronómico Hispano-Alemán (CAHA) at Calar Alto, operated jointly by the MaxPlanck Institut für Astronomie and the Instituto de Astrofísica de Andalucía (CSIC). observed (Watson \& Hanson 1997; Feldt et al. 2003; Alvarez et al. 2004; Bik et al. 2005), perhaps due to departures from the spherical symmetry giving rise to particular favorable viewing geometries. Such rare cases are of special value, since they provide the only views of the photospheres of $\mathrm{OB}$ stars at such early phases of their lives. On the other hand, visibility of the ionizing star may also indicate that the object is past the UCHIIR stage but still displays a spectral energy distribution leading to its classification as such, suggesting that the duration of the embedded phase may be overestimated if based on samples selected from far-infrared and radio properties alone.

G76.188+0.098 (=IRAS 20220+3728), located in the direction of the Cygnus X region (Wendker et al. 1991, and references therein), is a source with the typical far-infrared spectral energy distribution of an embedded massive star. Surveys at radio wavelengths have produced additional evidence supporting this classification (see Sect. 3). Earlier near-infrared imaging carried out by us (Comerón \& Torra 2001) revealed a small, elongated, very red source at the radio position with an unresolved central peak that was tentatively identified as the possible central star, although the limited spatial resolution of the observations presented in that work left some room for other interpretations. The new, higher-quality near-infrared observations presented in this paper show that the central source is a visual binary and offer important new insights on the nature of the object when combined with existing observations at other wavelengths. 


\section{Observations}

The observations presented in this paper were obtained with the Calar Alto $3.5 \mathrm{~m}$ telescope using the Omega-Cass near infrared camera and spectrograph, which uses a Rockwell $1024 \times$ 1024 pix $^{2}$ Hawaii HgCdTe array. Imaging observations were obtained on the night of 19 to 20 July 2005, and spectroscopy of the central source was further performed on the night of 24 to 25 September 2005. The reduction of all observations was carried out using standard tasks and dedicated scripts under IRAF ${ }^{1}$.

The images were obtained using camera optics providing a pixel scale of $0^{\prime \prime} 2 \mathrm{pix}^{-1}$. The excellent seeing conditions yielded stellar images with a full-width at half maximum of $0^{\prime \prime} 7$. The field was imaged through narrow-band filters $(\Delta \lambda / \lambda=0.01)$ centered at $2.166 \mu \mathrm{m}(\mathrm{Br} \gamma), 2.122 \mu \mathrm{m}\left(\mathrm{H}_{2} S(1) v=1 \rightarrow 0\right.$ transition), and $2.144 \mu \mathrm{m}$ ( $K$-band continuum). The narrowband images were composed of 9 frames obtained by slightly offsetting the telescope on a grid of $3 \times 3$ points $40^{\prime \prime}$ in side. An exposure of $120 \mathrm{~s}$ duration was obtained at each pointing, thus totalling $18 \mathrm{~min}$ of exposure time per filter. No separate sky frames were obtained, since the dither pattern amplitude exceeded the angular size of the HII region, thus allowing us to construct an appropriate sky frame by median filtering of the on-target images uncorrected for telescope offsets. The skysubtracted images were divided by flat field frames, registered using the numerous stellar images in each frame as a reference, and combined.

Spectroscopy of the central source was carried out using a grism covering the entire $K$ band with a resolution of $R=420$ ( 2 pixels) and a slit of $100 \mu \mathrm{m}$, corresponding to $0^{\prime \prime} 6$ on the sky. Pairs of spectra were obtained by integrating for $60 \mathrm{~s}$, then offsetting the telescope by $30^{\prime \prime}$ along the slit (which was oriented in the North-South direction), and integrating again for the same amount of time. Due to some technical difficulties with accurate pointing the star fell partly outside the slit in some of the frames, which were discarded. We retained 77 frames, corresponding to a total exposure time of $77 \mathrm{~min}$. Consecutive pairs of spectra obtained at different offset positions were subtracted from each other, and the stellar spectra were traced and extracted from such pairwise-subtracted frames. The G0V-type star HD194177, lying only $18^{\prime}$ apart on the sky, was observed in the same way at regular intervals and used for the subtraction of the telluric features from the spectra of the source of interest. Wavelength calibration of each frame was performed using the $\mathrm{OH}$ airglow spectrum as a built-in reference frame (Oliva \& Origlia 1992). The wavelength-calibrated spectra ratioed by the telluric reference star were then combined into a single spectrum. Approximate relative flux calibration was performed by multiplying this resulting spectrum by the spectrum of a black body at $T=5930 \mathrm{~K}$, expected to provide a good fit to the spectral energy distribution of HD194177 in the $K$ band.

\section{The G76.188+0.098 compact HII region}

\subsection{A summary of existing far-infrared and radio observations}

Although no detailed study of G76.188+0.098 in the near infrared has been published to date, the region has been included in multiple surveys at radio wavelengths. This has been largely motivated by its spectral energy distribution in the IRAS bands, in

${ }^{1}$ IRAF is distributed by NOAO, which is operated by the Association of Universities for Research in Astronomy, Inc., under contract to the National Science Foundation.
Table 1. Main data of G76.188+0.098.

\begin{tabular}{lc}
\hline \hline$\alpha(2000)^{1}$ & $20^{\mathrm{h}} 23^{\mathrm{m}} 44^{\mathrm{s}} 09$ \\
$\delta(2000)^{1}$ & $+37^{\circ} 38^{\prime} 10^{\prime \prime} 6$ \\
Size & $\simeq 15^{\prime \prime} \times 10^{\prime \prime}($ IR images $)$ \\
Radial velocity & $-2.7 \mathrm{~km} \mathrm{~s}^{-1}$ \\
$S_{v}(12 \mu \mathrm{m})^{3}$ & $11.1 \mathrm{Jy}$ \\
$S_{v}(25 \mu \mathrm{m})^{3}$ & $127 \mathrm{Jy}$ \\
$S_{v}(60 \mu \mathrm{m})^{3}$ & $947 \mathrm{Jy}$ \\
$S_{v}(100 \mu \mathrm{m})^{3}$ & $2131 \mathrm{Jy}$ \\
$S_{v}(2 \mathrm{~cm})^{4}$ & $347 \mathrm{mJy}$ \\
$S_{v}(6 \mathrm{~cm})^{4}$ & $362 \mathrm{mJy}$ \\
$S_{v}(20 \mathrm{~cm})^{5}$ & $417 \mathrm{mJy}$ \\
$S_{v}(91.7 \mathrm{~cm})^{6}$ & $534 \mathrm{mJy}$ \\
\hline
\end{tabular}

Notes:

1 Position of the central star. ${ }^{2}$ Bronfman et al. (1996). ${ }^{3}$ IRAS Point Source Catalog fluxes. ${ }^{4}$ Miralles et al. (1994). ${ }^{5}$ Zoonematkermani et al. (1990). ${ }^{6}$ Taylor et al. (1996).

which G76.188+0.098 appears as a bright source with extremely red colors between 12 and $60 \mu \mathrm{m}$ (see Table 1). The similarity of the IRAS spectral energy distributions of compact HII regions containing embedded massive stars, already remarked by Chini et al. (1987), was used by Wood \& Churchwell (1989) to define color-color criteria able to reliably select all UCHIIRs while virtually excluding any other possible sources contaminating the sample. Such color-color criteria, which have been widely used in numerous subsequent works to define samples of bona-fide UCHIIR candidates to be observed at other wavelengths, are fully met by G76.188+0.098.

High resolution VLA radio continuum maps at 2 and $3.6 \mathrm{~cm}$ presented by Kurtz et al. (1994), with a resolution of $0^{\prime \prime} 5$ and $0^{\prime \prime} 9$ respectively, show G76.188+0.098 as a multi-peaked compact source with an overall shell-like morphology approximately $12^{\prime \prime}$ across. The shell center is 7 " West of the nominal IRAS point source position, but given the size of the IRAS positional error ellipse (whose major axis lies approximately in the East-West position) such offset is not significant. Radio continuum observations at lower resolution have been presented by Miralles et al. (1994), who measured integrated fluxes of 347 and $362 \mathrm{mJy}$ at 2 and $6 \mathrm{~cm}$, respectively. A similar flux, $447 \mathrm{mJy}$, is measured by Hughes \& MacLeod (1994) at $6 \mathrm{~cm}$ with a beam of $3^{\prime \prime}$. Such fluxes are sensibly above those given by Kurtz et al. (1994) at $2 \mathrm{~cm}(67.6 \mathrm{mJy})$ and $3.6 \mathrm{~cm}$ (109.1 mJy), probably indicating substantial resolved extended emission. A comparison among the fluxes measured between $2 \mathrm{~cm}$ and $20 \mathrm{~cm}$ (Table 1) obtained with similar beam sizes of $\simeq 5^{\prime \prime}$ show no signs of a turnover frequency in that range ${ }^{2}$. The optically thin emission up to $20 \mathrm{~cm}(1.42 \mathrm{GHz})$ is in contrast with the higher turnover frequencies obtained for typical UCHIIR. Indeed, the emission measure derived from the radio continuum fluxes by Miralles et al. (1994), $E M=2.2 \times 10^{6} \mathrm{~cm}^{-6} \mathrm{pc}$, implies a turnover frequency around $\simeq 1 \mathrm{GHz}$ (Mezger \& Henderson 1967) and, when combined with the approximate size of the region, translates into an electron density that is low by UCHIIR standards (see Sect. 4).

2 The flux at $91.7 \mathrm{~cm}$ (Taylor et al. 1996) listed in Table 1 may suggest optically thin emission even at that wavelength, but the beam size used in that study is considerably larger $\left(1^{\prime}\right)$ and may include unrelated background sources. The same is true for the flux of $810 \mathrm{mJy}$ at $73.5 \mathrm{~cm}$ reported by Wendker et al. (1991), who used an even larger beam of $3^{\prime} 5 \times 5^{\prime} 2$. 


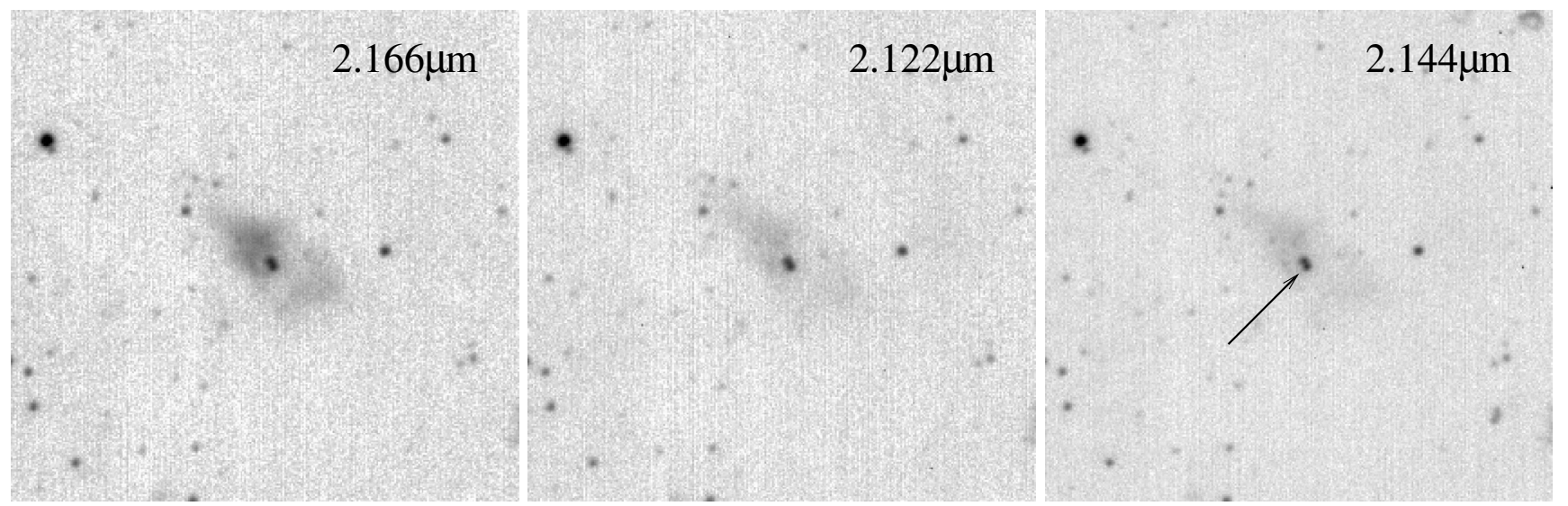

Fig. 1. G76.188+0.098 as seen through different narrow filters in the $K$ band. No continuum subtraction has been performed on the 2.166 (Brackett $\gamma$ ) or the $2.122 \mu \mathrm{m}\left(\mathrm{H}_{2} S(1) v=1 \rightarrow 0\right)$ images. The central binary system is indicated in the $K$-band continuum $(2.144 \mu \mathrm{m})$ image. The field is approximately $40^{\prime \prime} \times 40^{\prime \prime}$ with North to the top and East to the left.

Low-resolution ${ }^{13} \mathrm{CO}(J=1 \rightarrow 0)$ observations of the region surrounding G76.188+0.098 indicate that the HII region lies near the Southern edge of a larger molecular complex extending more than $20^{\prime}$ across the sky, which also hosts the compact HII region G76.175+0.127 (Casoli et al. 1986; Kurtz et al. 1994). G76.188+0.098 is also detected in other lines tracing high density gas such as $\mathrm{HCO}^{+}$(critical density $n_{\text {crit }} \simeq$ $10^{5} \mathrm{~cm}^{-3}$; Richards et al. 1987), CS $\left(n_{\text {crit }} \simeq 10^{4}-10^{5} \mathrm{~cm}^{-3}\right.$; Bronfman et al. 1996) and $\mathrm{NH}_{3}\left(n_{\text {crit }} \simeq 5 \times 10^{3} \mathrm{~cm}^{-3}\right.$; Miralles et al. 1994). The warm rotational temperature $\left(T_{R}=22 \mathrm{~K}\right.$; Miralles et al. 1994) derived from the latter molecule significantly exceeds the values around $T_{R} \sim 10 \mathrm{~K}$ usually found in quiescent clouds, most probably due to the proximity of the HII region. Water maser emission indicative of very high density gas $\left(n>10^{8} \mathrm{~cm}^{-3}\right)$ has been reported by Scalise et al. (1989) at a radial velocity $v_{\mathrm{LSR}}=-35.4 \mathrm{~km} \mathrm{~s}^{-1}$, much more negative than the velocities measured from the other molecular lines, which are all comprised in the $v_{\mathrm{LSR}}=-3$ to $0 \mathrm{~km} \mathrm{~s}^{-1}$ range. The water maser emission detection, carried out with a beam size of $3^{\prime} 7$ that prevents to pinpoint its precise origin in the high resolution radio or infrared observations, could not be confirmed by Miralles et al. (1994), perhaps due to variability. We also note that, although detection limits are not given in Scalise et al.'s (1989) paper, G76.188+0.098 has the faintest among their reported fluxes and is thus presumably very close to their detection threshold. If real, the large velocity difference may be due to an outflow, to which water masers are often associated. Additional evidence for a molecular outflow in G76.188+0.098, although at much lower velocities, has been recently presented by Zhang et al. (2005), who have mapped the region in the $\operatorname{CO}(J=2 \rightarrow 1)$ transition. The CO emission integrated over the entire region (see Fig. 2 of Zhang et al. 2005) displays a broad wing extending to $7 \mathrm{~km} \mathrm{~s}^{-1}$ bluewards from the emission peak, whereas the redshifted wing is narrower but displays a shoulder peaking at $v_{\mathrm{LSR}}=+1 \mathrm{~km} \mathrm{~s}^{-1}$ and extending to $v_{\mathrm{LSR}}=10 \mathrm{~km} \mathrm{~s}^{-1}$. The blueshifted emission is detected all over the area surrounding G76.188+0.098 and may actually be contributed by background gas, whose negative $v_{\mathrm{LSR}}$ is expected from the larger distance. In contrast, the redshifted gas is much more concentrated toward the position of G76.188+0.098.

We finally note that evidence for a HI expanding shell around G76.188+0.098, interpreted as the neutral atomic layer between the ionization and dissociation fronts bounding the expanding HII region, has been reported by Kothes \& Kerton (2002) appearing as a blueshifted absorption line, and possibly also a redshifted component, indicating an expansion velocity of $5.1 \mathrm{~km} \mathrm{~s}^{-1}$.

\subsection{New near-infrared imaging of the nebula}

The images obtained through the $\mathrm{Br} \gamma, \mathrm{H}_{2}$, and $K$-continuum filters are presented in Fig. 1. The nebula is well visible in all three images, including the $K$-continuum one, indicating a significant level of continuum emission perhaps by heated dust. On the other hand the nebula looks virtually identical in the images taken through both the $\mathrm{H}_{2}$ and $K$ continuum filters, suggesting that neither shocks in the molecular gas nor $\mathrm{H}_{2}$ excitation in the photodissociation region at the interface between the ionized gas and the molecular cloud contribute significantly to the emission, as confirmed by the spectra discussed in Sect. 3.3.

The appearance in the $\mathrm{Br} \gamma$ image is reminiscent of a bipolar nebula with an approaching northeastern lobe, an obscuring equatorial dust belt seen at an angle of $\sim 45^{\circ}$, and a less prominent, receding southwestern lobe. However, a comparison with the VLA radio maps (Kurtz et al. 1994; see Fig. 2) suggests a different interpretation. The "belt" separating the northern and southern "lobes" in the near-infrared images is also devoid of radio emission, indicating that the lack of infrared emission is real and not due to obscuration. The combination of the radio continuum maps and the near-infrared images rather suggests that the ionized gas is mainly distributed along a shell or perhaps a torus, which appears as a ring-shaped structure when projected on the sky. The ring is best delineated by the $3.6 \mathrm{~cm}$ map, but also traced by the near-infrared emission given the general agreement between the radio continuum contours and the shape of the nebula in $\operatorname{Br} \gamma$. The ring is disrupted at some locations, and its Southeastern edge seems to be completely missing. The near infrared emission in the northeastern lobe extends somewhat farther than the radio continuum emission, which might be due to an outflow of lower density gas parallel to the axis of the ring. The diameter of the ring seen in radio continuum emission is estimated to be $11^{\prime \prime}$.

\subsection{The central binary system}

Besides a detailed new view of the morphology of the nebula, the main contribution of the new near-infrared images to our knowledge of the region is the detection of a very red, almost point-like 


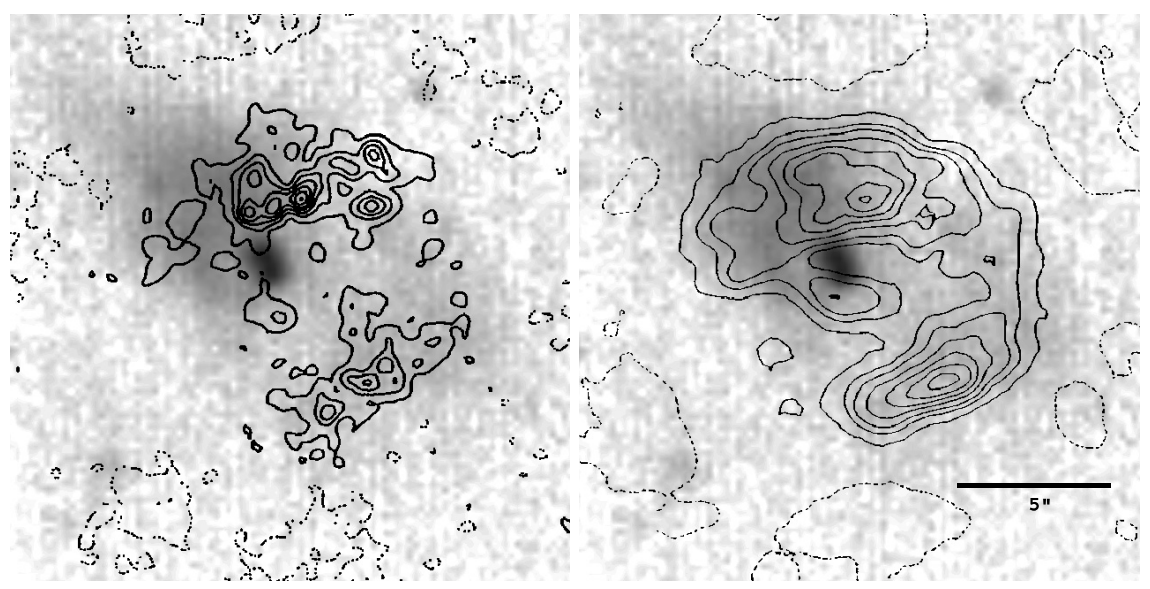

Fig. 2. Contours of the VLA images at $2 \mathrm{~cm}$ (left) and $3.6 \mathrm{~cm}$ (right) obtained by Kurtz et al. (1994) superimposed on our Bry image. Peak fluxes are $2.41 \mathrm{mJy} / \mathrm{beam}$ at $2 \mathrm{~cm}$ and $3.76 \mathrm{mJy} / \mathrm{beam}$ at $3.6 \mathrm{~cm}$. The synthesized beam $F W H M$ sizes are $0^{\prime \prime} 5$ and $0^{\prime \prime} 9$ at 2 and $3.6 \mathrm{~cm}$ respectively, and the plotted contours are separated at intervals of approximately $0.15 \mathrm{mJy} / \mathrm{beam}$. See Figs. 59 and 60 of Kurtz et al. (1994) for more details.

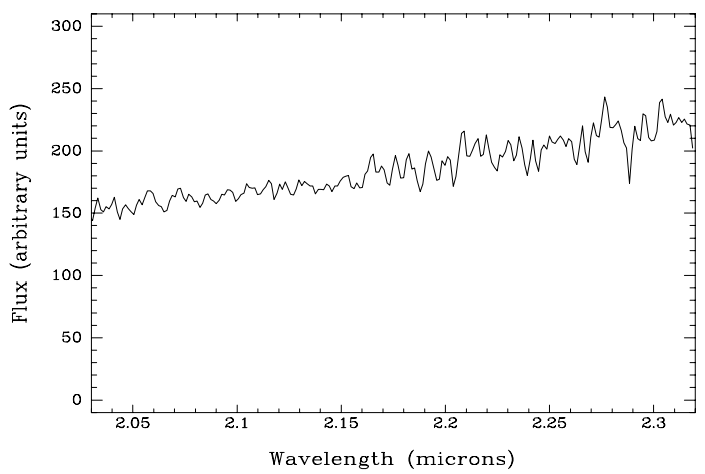

Fig. 3. Combined spectrum of the central stars of G76.188+0.098. The spectrum is featureless at the $\mathrm{S} / \mathrm{N}$ obtained in our observations, as expected from a binary system composed by two O-type stars.

object near the center of the near-infrared nebulosity and inside the ring-like structure described in Sect. 3.2. The good quality of our images allows one to see that the central object is actually resolved into a binary system of two point sources, with components of similar brightness and reddening separated by approximately 0 " 5 .

Photometry of the system in the $K$ continuum image, taking the 2MASS magnitudes of brighter, isolated stars in the field as a zero point reference, indicates $K$ magnitudes of $K_{\mathrm{S}}=13.8$ and $K_{\mathrm{S}}=14.1$ for the brightest and faintest member of the system respectively. The $H-K_{\mathrm{S}}$ color is similar for both, $H-K_{\mathrm{S}}=2.4$. The combined luminosity of the central binary is below that implied by $K_{\mathrm{S}}=12.236$ listed in the 2MASS Point Source Catalogue, which in turn is similar to that measured by Comerón \& Torra (2001). This is almost certainly due to the contribution of the compact HII region, which can be separated only now thanks to the superior resolution of our images. The $K$-band spectrum of the central object that we obtained is actually the merged spectrum of both components, due to the less optimal seeing under which our spectroscopic observations were obtained. This spectrum, shown in Fig. 3, is featureless at the signal-to-noise achieved, as expected from an OB-type star. A more accurate determination of the spectral type is not possible at the moment, since most of the strongest absorption features used for classification of early-type stars (Hanson et al. 1996) have equivalent widths $<1 \AA$, which is about three times below our detection limit. However, the lack of $\mathrm{CO}$ absorption

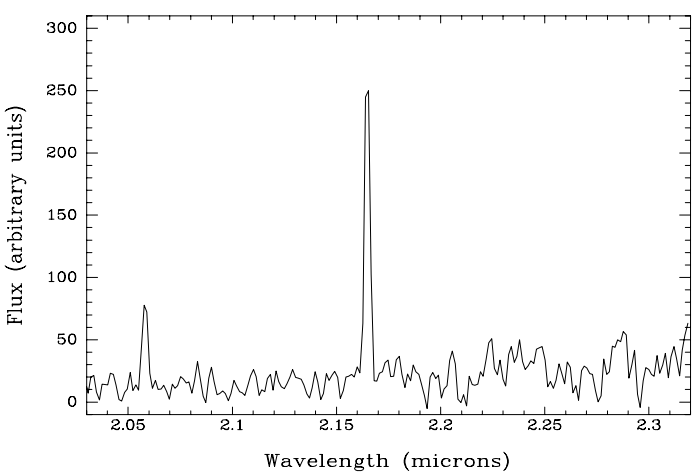

Fig. 4. Spectrum of the G76.188+0.098 nebula in the immediate vicinity of the central binary system, showing the prominent emission at $\mathrm{HeI}(2.058 \mu \mathrm{m})$ and $\mathrm{Br} \gamma(2.166 \mu \mathrm{m})$.

at $\lambda>2.29 \mu \mathrm{m}$ and the absence of other features, as well as the shape of the continuum, are sufficient to show that the central object is not composed of cool stars. We will thus consider that the binary system is the central source of the region and the responsible for its ionization. Taking into account the small range of intrinsic infrared colors displayed by $\mathrm{OB}$ stars in the infrared (e.g. Tokunaga et al. 2000), and assuming that all the reddening is produced by foreground extinction, the measured $H-K_{\mathrm{S}}$ color leads to an estimated extinction $A_{K}=4.4 \pm 0.1$ (Rieke \& Lebofsky 1985).

\subsection{The spectrum of the HII region and its implications on the ionizing stars}

The spectrum of the nebula presented in Fig. 4 can be used to further constrain the spectral type of the components of the central system despite the fact that only two emission lines, $\mathrm{HeI}(2.058 \mu \mathrm{m})$ and $\mathrm{Br} \gamma$, are visible. Their measured flux ratio is $0.26 \pm 0.03$, or 0.38 after correcting for the wavelengthdependent extinction at both wavelengths using $A_{K}=4.4$ and $A_{\lambda} \propto \lambda^{-1.75}$. The behavior of the $\mathrm{HeI}(2.058 \mu \mathrm{m}) / \mathrm{Br} \gamma$ ratio as a function of the temperature of the central star, and other parameters such as the density and the size of the central cavity of the nebula, has been discussed in detail by Lumsden et al. (2003). In their models, these authors predict an intrinsic ratio peaking at a value larger than the one that we measure by a factor $2-3$ at a stellar temperature $T_{*} \simeq 36000 \mathrm{~K}$. The much smaller value that 
we measure is in better agreement with those expected when the temperature of the ionizing source is either significantly above or below this value in the stellar models used by Lumsden et al. (2003). However, these temperatures must be taken with caution as the line ratio is mainly determined by the hydrogenionizing $\left(Q_{0}\right)$ and helium-ionizing $\left(Q_{1}\right)$ fluxes of the star, rather than by its photospheric temperature. Possible systematic effects resulting from shortcomings of the model atmospheres used in their calculations are noted by Lumsden et al. (2003).

To provide a better estimate of the parameters of the central stars as constrained by the nebula models, we have used the recent temperature- $\log q_{0}$ and temperature- $\log q_{1}$ relations from Martins et al. (2005), where $q_{0}=Q_{0} /\left(4 \pi R_{*}^{2}\right)$, $q_{1}=Q_{1} /\left(4 \pi R_{*}^{2}\right)$, and $R_{*}$ is the radius of the star. We prefer not to use the values of $Q_{0}$ and $Q_{1}$ given by Martins et al., since the stellar radii that they use are based on an empirical $M_{V}$-spectral type calibration derived from emerged $\mathrm{O}$ stars that are likely to be more evolved on the average than ZAMS stars of the same temperature, as those authors note. The difference is important over virtually the entire range of $\mathrm{O}$ spectral types and increases towards later types, as can be seen in Fig. 12 of Martins et al. (2005). Therefore, we have recomputed the $Q_{0}$ and $Q_{1}$ obtained from the models of Martins et al. but using the stellar radii at the ZAMS in the CoStar models of Schaerer et al. (1996a, 1996b). Similarly, we have recalculated in a similar way the absolute magnitudes $M_{V}$ given by Martins et al. (2005) to account for the smaller radii that we adopt.

With this choice of calibrations, which uses the most upto-date model atmospheres of $\mathrm{O}$ stars in combination with the ZAMS models that are expected to describe in a more adequately way the global properties of the central stars of compact HII regions, we find that the $Q_{0}$ and $Q_{1}$ values that reproduce the observed $\mathrm{HeI}(2.058 \mu \mathrm{m}) / \mathrm{Br} \gamma$ ratio correspond to stellar temperatures of either approximately $T_{*} \simeq 33000 \mathrm{~K}$, or $T_{*}>42000 \mathrm{~K}$, which correspond to the spectral types O9 ("late-type" solution) or earlier than $\mathrm{O} 4.5$ ("early-type" solution), respectively. It should be noted that both $Q_{0}$ and $Q_{1}$ quickly drop as the absolute magnitude becomes fainter, implying that the contribution to the ionizing flux of the nebula is in either case dominated by the brighter component of the binary system.

In the late-type solution, the corresponding absolute magnitude of the primary component of the central binary system is $M_{V}=-3.3$, of $M_{K}=-2.5$ taking into account the intrinsic $V-K$ color of a O9 star (Lejeune \& Schaerer 2001). The steep dependence of $\operatorname{HeI}(2.058 \mu \mathrm{m}) / \mathrm{Br} \gamma$ near $T_{*} \simeq 33000 \mathrm{~K}$ limits the uncertainty in $T_{*}$ to about $1000 \mathrm{~K}$, corresponding to an uncertainty of 0.5 subclasses in the spectral type and 0.2 mag in the absolute magnitude. The absolute magnitude difference implies in turn that the companion has a B0 spectral type. The distance that we derive is thus $D=2.4 \pm 0.3 \mathrm{kpc}$, which is considerably closer than the kinematical distance adopted in previous studies of G76.188+0.098. Nevertheless, it is well known that the shallow dependence of the radial velocity with the distance in the direction of Cygnus makes the radial velocity virtually useless for the derivation of distances up to about $5 \mathrm{kpc}$ from the Sun. Therefore, we do not consider this discrepancy as meaningful. On the other hand, the combined value of $\log Q_{0}$ that we find for this solution by adding the ionizing flux from both components, $\log Q_{0}\left(\mathrm{~s}^{-1}\right)=47.9$, is remarkably close to the independent estimate obtained from the radio continuum flux by Miralles et al. (1994), for which we obtain essentially the same quantity after correcting the value given by Miralles et al. for the difference between their assumed distance and the distance that we derive above.
The early-type solution implies $M_{K}<-3.6$ for the primary star and a distance in excess of $4 \mathrm{kpc}$, which is still consistent with the distance constraints set by the radial velocity of the nebula. However, in this case the ionizing flux is above that inferred from the radio continuum by at least one order of magnitude, which would imply that the compact HII region is densitybounded in most directions ${ }^{3}$. While this is not ruled out by the observations and is not an implausible scenario, the good match obtained between the independent estimates of $Q_{0}$ in the latetype case, and the expectation that the HII region hosting such a deeply embedded binary system is ionization-bounded, leads us to strongly prefer the late-type solution as the one representing the actual case of G76.188+0.098.

The bolometric luminosity of a central system composed of two ZAMS stars with spectral types $\mathrm{O} 9$ and $\mathrm{B} 0$ is $\log L \simeq 4.7$ (Schaerer et al. 1996a,b). This can be compared to the total infrared luminosity of the nebula, for which we obtain $\log L_{\mathrm{IR}} \simeq$ 4.2 from the total infrared flux estimate based on the IRAS band measurements (Casoli et al. 1986) and the distance $D=2.4 \mathrm{kpc}$ as discussed above. Both fluxes should coincide if the entire contribution to the luminosity of the nebula arose from the central binary alone, and assuming that the luminosity output of the central source is entirely reprocessed and emitted in the far infrared by dust in the nebula (Churchwell 1990). The agreement within a factor of 3 that we find may be considered as fair given the uncertainties in the estimate of both quantities. The discrepancy between both luminosities increases if we adopt the early-type solution, as the increase in $L_{\mathrm{IR}}$ due to the greater distance is offset by an even greater increase of the bolometric luminosity of the central system.

\section{Discussion}

Applying the classical IRAS flux-based selection criteria (Wood \& Churchwell 1989; Codella et al. 1994), expected to produce largely uncontaminated samples, G76.188+0.098 has been classified as a UCHIIR containing an embedded late-O or early-B star. Radio continuum maps show indeed a compact ring-like structure described by Hanson et al. (2002) as a fascinating multilobed morphology, almost like a detached shell, and millimeter observations probing dense molecular gas confirm the existence of the warm dense cores that are frequently found in the vicinity of massive embedded stars. Except for the lack of a high turnover frequency in the radio continuum spectrum, G76.188+0.098 thus seems to qualify as a bona fide UCHIIR. The discovery of a binary star with a $0^{\prime \prime} 5$ separation at its center provides a way to constrain additional characteristics of the nebula, as well as a unique insight on the earliest stages of evolution of an embedded massive binary.

The combination of the existing observations presented in this paper shows that the central binary lies roughly at the center of an incomplete, elongated ring-like structure, in a zone that is

${ }^{3}$ The fact that our small $\mathrm{HeI}(2.058 \mu \mathrm{m}) / \mathrm{Br} \gamma$ is well below the ratios $(0.8-1.2)$ measured by Lumsden et al. (2003) in UCHIIRs excited by early- and mid O-type stars might be interpreted as evidence against the early type solution. However, the systematic discrepancy between measured ratios and model predictions for the earliest spectral types has been attributed by Lumsden et al. (2003) to a higher rate of collisional excitation of the HeI $2^{1} P$ level than the models account for in the high density medium of typical UCHIIRs. This explanation may not apply to G76.188+0.098 however, since its electron density seems to be considerably smaller than that of the typical UCHIIRs included in Lumsden et al.'s (2003) sample, as derived by Miralles et al. (1994; see Sect. 3.1). 
fairly devoid of ionized gas. At our adopted distance for the region, the size measured in the infrared images (see Table 1) corresponds to a physical size of $0.17 \mathrm{pc} \times 0.12 \mathrm{pc}$, which is near the borderline separating compact from ultracompact HII regions. The relatively large size of the region, together with the also large size of the central cavity, the broken appearance of the radio contours, the rather low average density ${ }^{4}$ of $9.2 \times 10^{3} \mathrm{~cm}^{-3}$, and the moderate extinction towards the embedded source for UCHIIR standards, leads us to propose that G76.188+0.098 is a HII region near the end of the ultracompact stage in which the central object, in this case a binary system with components of derived spectral types $\mathrm{O} 9$ and $\mathrm{B} 0$ with a projected separation of $1200 \mathrm{AU}$, is beginning to emerge from the embedded phase making itself accessible to near-infrared observations.

As discussed in Sect. 3.4, the existing observations and those presented here do not exclude the possibility that G76.188+0.098 may actually be a more distant region, lying between 4 and $5 \mathrm{kpc}$ from the Sun, ionized by very early stars. Such possibility is allowed by the radial velocity measurements but, as explained above, its main implication would be that most of the ionizing flux escapes the nebula, which would be inconsistent with its UCHIIR classification. Should future evidence support the early-type solution against the late-type solution that we clearly favor here, a very different scenario should be developed in order to account for all the observed features. In this regard, the alternative could be to interpret the ionized region as the inner edge of an extended, dense slab being eroded by the central binary producing a flow of gas away from it, while most of the ionizing flux of the central object would escape unimpeded in the directions not intercepted by the slab. We do not further explore this possibility here, since we consider it to be rather unlikely in view of the good agreement provided by the late-type solution.

The possible association of G76.188+0.098 with other large structures in Cygnus, which might provide an independent assessment of its distance, cannot be unambiguously established. The region is projected within the contours of Cygnus X, an extended region of the sky containing multiple sites of recent star formation (e.g. Wendker et al. (1991) and references therein) and is located in projection between two well studied objects, the bipolar nebula S106 and the complex ON2 (see Schneider et al. (2002) and Comerón \& Torra (2001) respectively for summaries of observations of each of these regions). A physical relationship with either region seems unlikely, though. The distance of S106 is likely to be as close as 600 pc (Schneider et al. 2002), while the distance of ON2 is reasonably well determined at $900 \mathrm{pc}$ thanks to its association with the Berkeley 87 cluster (Turner \& Forbes 1982). Such short distances can be reliably ruled out for $\mathrm{G} 76.188+0.098$, as they would imply a fainter absolute magnitude of the central binary by almost 2 mag and components of mid-B spectral types, by far insufficient to produce neither the ionizing radiation required by the radio observations nor the observed intensity of the HeI $2.058 \mu \mathrm{m}$ line. The same reasoning may be used to rule out a physical relationship with Cygnus OB1, one of the numerous OB associations in that general direction of the galactic disk, on which G76.188+0.098 is projected, given the estimated distance of $1.3 \mathrm{kpc}$ to Cygnus OB1 (Garmany \& Stencel 1992). We note however that many structures in that region of the galactic disk, which is dominated by the rich association Cygnus OB2, appear to be physically connected as shown by recent molecular-line

\footnotetext{
${ }^{4}$ Value calculated from the radio continuum flux at $6 \mathrm{~cm}$ given by Miralles et al. (1994), taking into account the actual size of the HII region and correcting for the adopted distance of $2.4 \mathrm{kpc}$.
}

and thermal infrared observations presented by Schneider et al. (2006). The distance of Cygnus OB2 itself is still somewhat controversial, but values between $1.4 \mathrm{kpc}$ (Hanson 2003) and $1.8 \mathrm{kpc}$ (Massey \& Thompson 1991) are likely and marginally consistent within the uncertainties with our estimated distance of G76.188+0.096, which might thus be a star forming site in this vast complex.

\section{Summary}

In this paper we have reported new near-infrared observations of G76.188+0.098, a candidate ultracompact HII region (UCHIIR) in Cygnus. The nebula is well seen in the $2 \mu \mathrm{m}$ region, where its structure roughly outlines the broken ring morphology mapped in the radio continuum. The most important result of this study is the detection of the central exciting source, which is actually a binary system obscured by an extinction of $A_{K} \simeq 4.4 \mathrm{mag}$, or $A_{V} \simeq 40$ in the visible and whose components are separated by $0^{\prime \prime} 5$. The $K$-band emission-line spectrum of the nebula shows a low value of the $\operatorname{HeI}(2.058 \mu \mathrm{m}) / \operatorname{Br} \gamma$ ratio, which we use to constrain the spectral types of the central stars. We identify two possible solutions, a first one that yields a $\mathrm{O} 9+\mathrm{B} 0$ central binary at a distance of $2.4 \pm 0.3 \mathrm{kpc}$ from the Sun, and a second one that places the region beyond $4 \mathrm{kpc}$ with a spectral type $\mathrm{O} 4.5$ or earlier of the main component of the binary. We strongly favor the first interpretation, as it produces a very good match between the expected ionizing flux of a $\mathrm{O} 9+\mathrm{B} 0$ central binary system and the ionizing flux derived from the radio continuum flux of the nebula, while the second one would imply that the HII region is density-, rather than ionization-bounded.

Several lines of evidence suggest that G76.188+0.098 is a HII region near the end of the ultracompact phase. The central object has already carved a large central cavity of about $0.06 \mathrm{pc}$ radius, and the shell-like structure from where the HII emission arises seems heavily eroded at many places and with its southeastern portion missing. The electron density is low for UCHIIR standards, as already inferred from the absence of a high-frequency turnover of the radio continuum spectral index. Finally, the size is near the upper edge that characterizes the ultracompact stage. The fact that the central binary has become easily visible in the near-infrared also indicates a line-ofsight extinction well below those typically estimated for young UCHIIR, implying that it is close to reaching the end of the embedded phase.

G76.188+0.098 thus seems to provide one more valuable example to the handful of cases in which the central star of a UCHIIR has been detected, and it is to our knowledge the first instance in which the central system turns out to be a detached high-mass binary, thus providing an attractive example of a massive binary system in its earliest evolutionary stages.

Acknowledgements. It is a pleasure to thank one more time the staff of the Calar Alto observatory for their assistance during their observations, most especially Dr. Nicolás Cardiel and Mr. Jesús Aceituno for their efforts to overcome technical difficulties encountered with Omega-Cass during our July 2005 run. Dr. Roland Gredel, Director of the Calar Alto observatory, is thanked for the allocation of Director's Discretionary Time (DDT) to the completion of this project. The careful management and execution of the DDT run in September 2005 by Mr. Ulrich Thiele, Mr. Jesús Aceituno, and Mr. Santos Pedraz is also greatly appreciated. We are particularly thankful to the referee, whose comments on the first version of this paper prompted a reassessment of the properties of the central binary system that resulted in a substantial reinterpretation of our results. J.T. acknowledges support by the Spanish Ministry of Science and Technology under contract AYA2003-07736. This research has made use of data products from the Two Micron All Sky Survey, which is a joint project of the University of Massachusetts and the Infrared Processing and Analysis 
Center/California Institute of Technology, funded by the National Aeronautics and Space Administration and the National Science Foundation

\section{References}

Alvarez, C., Feldt, M., Henning, Th., et al. 2004, ApJS, 155, 123 Bik, A., Kaper, L., Hanson, M. M., \& Smits, M. 2005, A\&A, 440,121 Bronfman, L., Nyman, L.Casoli, F., Dupraz, C., Gerin, M., Combes, F., \& Boulanger, F. 1986, A\&A, 169, 281

Chini, R., Krügel, E., \& Wargau, W. 1987, A\&A, 181, 378

Churchwell, E. 1990, A\&AR, 2, 79

Churchwell, E. 2002, ARA\&A, 40, 27

Codella, C., Felli, M., \& Natale, V. 1994, A\&A, 284, 233

Comerón, F., \& Torra, J. 2001, A\&A, 375, 539

Faison, M., Churchwell, E., Hofner, P., et al. 1998, ApJ, 500, 280

Feldt, M., Puga, E., Lenzen, R., et al. 2003, ApJ, 599, L91

Garmany, C. D., \& Stencel, R. E. 1992, A\&AS, 94, 211

Hanson, M. M. 2003, ApJ, 597, 957

Hanson, M. M., Conti, P. S., \& Rieke, M. J. 1996, ApJS, 107, 281

Hanson, M. M., Luhman, K. L., \& Rieke, G. H. 2002, ApJS, 138, 35

Hughes, V. A., \& MacLeod, G. C. 1994, ApJ, 427, 857

Kothes, R., \& Kerton, C. R. 2002, A\&A, 390, 337

Kurtz, S., Churchwell, E., \& Wood, D. O. S. 1994, ApJS, 91, 659

Lejeune, T., \& Schaerer, D. 2001, A\&A, 366, 538

Lumsden, S. L., Puxley, P. J., Hoare, M. G., Moore, T. J. T., \& Ridge, N. A. 2003 , MNRAS, 340, 799
Martins, F., Schaerer, D., \& Hillier, D. J. 2005, A\&A, 436, 1049 Massey, P., \& Thompson, A. B. 1991, AJ, 101, 1408

Mezger, P. G., \& Henderson, A. P. 1967, ApJ, 147, 471

Miralles, M. P., Rodríguez, L. F., \& Scalise, E. 1994, ApJS, 92, 173

Molinari, S., Brand, J., Cesaroni, R., \& Palla, F. 1996, A\&A, 308, 573

Oliva, E., \& Origlia, L. 1992, A\&A, 254, 466

Richards, P. J., Little, L. T., Toriseva, M., \& Heaton, B. D. 1987, MNRAS, 228, 43

Rieke, G. H., \& Lebofsky, M. J. 1985, ApJ, 288, 618

Scalise, E., Rodríguez, L. F., \& Mendoza-Torres, E. 1989, A\&A, 221, 105

Schaerer, D., de Koter, A., Schmutz, W., \& Maeder, A. 1996a, A\&A, 312, 475

Schaerer, D., de Koter, A., Schmutz, W., \& Maeder, A. 1996b, A\&A, 310, 837

Schneider, N., Simon, R., Kramer, C., Stutzki, J., \& Bontemps, S. 2002, A\&A, 384,225

Schneider, N., Bontemps, S., Simon, R., et al. 2006, A\&A, in press

Shepherd, D. S., \& Churchwell, E. 1996, ApJ, 457, 267

Taylor, A. R., Goss, W. M., Coleman, P. H., van Leeuwen, J., \& Wallace, B. J. 1996, ApJS, 107, 239

Tokunaga, A. T. 2000, in Allen's Astrophysical Quantities, ed. A. N. Cox (AIP Press)

Turner, D. G., \& Forbes, D. 1982, PASP, 94, 789

Watson, A. M., \& Hanson, M. M. 1997, ApJ, 490, L165

Wendker, H. J., Higgs, L. A., \& Landecker, T. L. 1991, A\&A, 241, 551

Wood, D. O. S., \& Churchwell, E. 1989, ApJS, 69, 831

Zhang, Q., Hunter, T. R., Brand, J., et al. 2005, ApJ, 625, 864

Zoonematkermani, S., Helfand, D. J., Becker, R. H., White, R. L., \& Perley, R. A. 1990, ApJS, 74, 181 